\title{
Prophylactic cranial irradiation in small-cell lung cancer: update on patient selection, efficacy and outcomes
}

This article was published in the following Dove Press journal:

Lung Cancer:Targets and Therapy

\author{
Farkhad Manapov ${ }^{1,2}$ \\ Lukas Käsmann' \\ Olarn Roengvoraphoj' \\ Maurice Dantes' \\ Nina-Sophie \\ Schmidt-Hegemann' \\ Claus Belka ${ }^{1,2}$ \\ Chukwuka Eze' \\ 'Department of Radiation Oncology, \\ University Hospital, LMU Munich, \\ Munich, Germany; ${ }^{2}$ Comprehensive \\ Pneumology Center Munich (CPC-M), \\ Munich, Germany
}

\begin{abstract}
Over $10 \%$ of small-cell lung cancer (SCLC) patients have brain metastases (BM) at initial diagnosis; more than 50\% will develop BM within 2 years. BM are detected in up to $80 \%$ of all patients at autopsy. After primary treatment, prophylactic cranial irradiation (PCI) has been established as standard of care in SCLC patients responding to initial therapy. Based on level I evidence, PCI significantly decreases the risk of intracranial relapse and shows a modest survival benefit after 3 years. However, the role of PCI in defined patient subgroups such as resected SCLC, elderly and extensive stage patients with access to magnetic resonance imaging surveillance and stereotactic radiotherapy is yet to be fully clarified. Furthermore, strategies to effective prevention of neurocognitive decline after PCI remain unclear. All these factors significantly impact treatment decision making and should be evaluated in prospective settings. New concepts such as hippocampal avoidance and drug neuroprotection prevent chronic neurocognitive effects reducing treatment-related side effects of PCI. The aim of this review is to present a summary and update of the latest evidence for patient selection, efficacy and outcome of PCI.
\end{abstract}

Keywords: PCI, small-cell lung cancer, treatment, patient selection, neurocognition

\section{Introduction}

Small-cell lung cancer (SCLC) is an aggressive type of cancer associated with poor prognosis due to rapid growth and early distant and loco-regional dissemination. ${ }^{1,2}$ Combined modality treatment, consisting of chemotherapy and thoracic radiotherapy (TRT) delivered concurrently or sequentially, is the standard of care for primary disease. ${ }^{3}$ Patients who respond to initial therapy without developing symptomatic brain relapse will be treated with prophylactic cranial irradiation (PCI). ${ }^{4-6}$ In an earlier study in extensive stage (ES)-SCLC, PCI significantly reduced the incidence of symptomatic brain metastases (BM) by approximately $25 \%$, which translated into improvement in median overall survival. ${ }^{6}$

Patients with limited stage (LS) achieve a median survival of 16-20 months and a 5 -year survival rate of $10 \%-20 \%$. The administration of PCI has been investigated in several studies and has been proven to be an independent prognostic factor. ${ }^{6-8}$ In 2008 , a small retrospective study first reported about the role of repeat contrast-enhanced cranial MRI immediately before the start of PCI for detection of occult intracranial relapse in patients who completed chemoradiotherapy. ${ }^{9}$ A randomized Phase 3 study by Le Péchoux et al established PCI with a total dose of 25 Gy delivered in 10 once-
Correspondence: Farkhad Manapov Department of Radiation Oncology, University Hospital, LMU Munich, Marchioninistrasse 15, Munich 81377, Germany

Tel +4989440057561

Fax +49 89440057562

Email Farkhad.Manapov@med.unimuenchen.de 
daily fractions as standard of care due to significant increase in mortality in the higher dose arm of $36 \mathrm{~Gy} .{ }^{10}$ Interestingly, a pooled analysis by the North Central Cancer Group revealed a survival difference between PCI total dose of 25 and 30 Gy probably associated with higher rates of adverse events in the 30 Gy group. ${ }^{11}$ Other prognostic factors such as age, sex, performance status and response to initial treatment were also reported to have an impact on patient outcome. ${ }^{12-16}$

Following 2 previous publications by Slotman et al, chemotherapy followed by TRT and PCI in treatment responders was established as the new treatment standard for ES disease. ${ }^{6,17}$ However, a recent Phase 3 study conducted by Takahashi et al at 47 institutions in Japan included an active MRI surveillance program before and after PCI and could not confirm a survival benefit of PCI in ES disease with confirmed absence of BM. ${ }^{18}$ However, TRT was not an obligatory part of multimodal treatment in the study and has to be taken into account when interpreting the data.

Although the delivery of PCI in both LS- and ES-SCLC is considered to be well established, treatment-related side effects need further extensive investigation. PCI is known to be associated with acute side effects including alopecia, nausea, headache, fatigue as well as chronic neurologic sequelae such as decline in neurocognitive function. ${ }^{19} \mathrm{Sev}-$ eral strategies such as hippocampal avoidance and/or drug neuroprotection are currently being investigated in order to limit potential side effects. ${ }^{20-23}$

The aim of this review is to present a summary of the latest evidence for various patient subgroups, efficacy and outcome of PCI considering new technical and imaging opportunities.

\section{$\mathrm{PCl}$ with hippocampal avoidance}

Patient concern regarding neurotoxicity is the most common reason for PCI omission. ${ }^{23}$ Memory decline, changes in appetite, nausea, and vomiting as well as hair loss are a major treatment concern.

However, the diagnosis of SCLC generally appears to negatively impact health-related quality of life (QOL) in comparison to the normal population in several functions such as daily activity, physical functioning, cognitive and emotional functioning. ${ }^{24} \mathrm{~A}$ systematic review of literature published in 2017 described that the impact on health-related QOL may be least in both LS- and ES-SCLC patients who responded to treatment, and greatest in ES patients who were treatment naïve. ${ }^{24}$ Importantly, QOL measured by patient-reported QOL scores after PCI showed a significant decline in QOL for up to 3 months after the completion of treatment. ${ }^{7}$
Prospective studies assessing QOL of patients in observation vs. PCI groups after initial treatment response are inconclusive. In the study by Le Péchoux et al, QOL was evaluated before PCI and routinely during follow-up up till 3 years. PCI was associated with mild deterioration of communication deficit, weakness of legs, intellectual deficit and memory dysfunction. ${ }^{10}$ However, further analyses reported that PCI was associated with a decline in Hopkins Verbal Learning Test-Revised (HVLT-R) and self-reported cognitive functioning. Additionally, due to the increased risk of developing chronic neurotoxicity in patients with $36 \mathrm{~Gy}$, a total PCI dose of 25 Gy remains the standard of care for patients with LS-SCLC patients attaining a complete response to initial chemoradiotherapy. ${ }^{10,25}$

A randomized Phase 3 trial of PCI in patients with locally advanced non-small-cell lung cancer did not find any significant differences in global cognitive function or QOL after PCI, but there was a significant decline in memory HVLT at 1 year. ${ }^{26}$

Importantly, a study by Simó et al revealed neuropsychological deficits together with notable brain-specific structural changes after chemotherapy and PCI, suggesting that chemotherapy and especially PCI are associated with the development of cognitive and structural brain toxic effects. ${ }^{27}$ Another study detected different markers of neuronal injury after PCI. These cerebrospinal markers such as neurofilament, T-tau or the levels of secreted amyloid precursor protein- $\alpha$ and $-\beta$ could potentially be used to assess the individual risk of developing long-term symptoms of chronic encephalopathy after PCI ${ }^{28}$ However, prospective larger trials with robust neurocognitive assessments and longer follow-up periods are needed to confirm these results.

In order to prevent neurocognitive decline, important brain regions such as the limbic circuit and hippocampal formation should be identified as organs/regions at risk. These regions are in reasonable suspicion to be responsible for memory and higher neurocognitive function and are mainly represented in the hippocampal region. The incidence of BM in the perihippocampal region has not been well investigated. Only limited information is available and earlier studies show that metastasis incidence in the perihippocampal region can vary from $4 \%$ to $27 \%$. $^{20,21,29}$

Earlier studies estimated the perihippocampal metastasis risk at $8.6 \%$ and claimed safety of hippocampal avoidance during whole-brain radiotherapy (HA-WBRT) for clinical testing. ${ }^{21}$ However, in other studies focusing on hippocampal metastasis, Korkmaz et al reported a hippocampal metastasis rate of $32 \%$ and questioned the usage of HA-WBRT. The 
prospective single-arm Phase-2 study (RTOG 0933) revealed that conformal avoidance of the hippocampus during wholebrain radiotherapy (WBRT) is associated with preservation of memory using the HVLT-R and QOL. ${ }^{22}$ Unfortunately, the median survival time was short (less than 7 months) and the assessments were performed from baseline up to 6 months.

Another small prospective study by Redmond et al showed a median follow-up time of 16.7 months and a 2-yearsurvival rate of $88 \% .{ }^{20}$ Interestingly, there was no significant neurocognitive performance decline between baseline and 6 or 12 months for any of the tests. Only two patients developed metastases in the underdosed region.

However, the patient collective was limited $(n=20)$ and larger studies should determine the potential benefit of hippocampal sparing.

Modern radiation techniques such as intensity-modulated radiotherapy permit administration of high radiation doses with avoidance of the perihippocampal regions. Nevertheless, the potential benefit of hippocampal sparing in limiting the neurocognitive decline caused by brain irradiation must be questioned by an increase of failure in the spared regions. Additionally, the potential survival benefit of PCI is important, but maintaining QOL by avoiding the physical and neurocognitive complications should be critically discussed with the patient in order to enhance shared decisionmaking. ${ }^{30}$ Currently, a host of randomized Phase $2 / 3$ trials is underway to clarify this benefit including cognitive and QOL assessment (NCT02736916, NCT02906384/ZJCH-HA-PCI, NCT02397733, NCT01780675/M12PHA, NCT02635009/ NRG-CC003 trials)

In summary, HA-WBRT could prevent neurocognitive decline, but the incidence of metastases in perihippocampal regions after PCI should be critically evaluated. The results of ongoing Phase 3 trials are highly warranted to confirm the findings from smaller studies to fully endorse the routine usage of hippocampus avoidance in clinical practice.

\section{$\mathrm{PCl}$ und drug neuroprotection}

There are several substances in preclinical studies that have demonstrated a positive effect on neuroprotection. However, till date, only a few substances have been tested within the ramifications of a Phase 3 study. The RTOG 0614 trial randomized 554 patients to receive placebo or the N-methyl-D-aspartate receptor antagonist memantine $(20 \mathrm{mg} / \mathrm{d})$ as a neuroprotectant, within 3 days of initiating radiotherapy for 24 weeks.

Memantine was well tolerated with a similar toxicity profile vs. placebo. In the memantine arm, there were fewer declines in delayed recall at 24 weeks, but the difference was non-significant. The memantine arm had significantly longer time to cognitive decline; the probability of cognitive function failure at 24 weeks was $53.8 \%$ vs. $64.9 \%$ in the memantine vs. placebo arm. Superior results were seen in the memantine arm for executive function at 8 and 16 weeks and for processing speed and delayed recognition at 24 weeks. ${ }^{31}$ Although the primary endpoint was technically non-significant, the updated NCCN guidelines for management of SCLC recommend considering memantine during and after administration of PCI.

In addition, donepezil, a neurotransmitter modulator was also evaluated in a Phase 3 placebo-controlled trial that randomized 198 brain tumor survivors $\geq 6$ months after partial- or whole-brain irradiation of which $8 \%$ underwent PCI to receive a single daily dose ( $5 \mathrm{mg}$ for 6 weeks, $10 \mathrm{mg}$ for 18 weeks) of donepezil or placebo. The authors concluded that treatment with donepezil did not significantly improve the overall composite score (primary endpoint), but it did result in modest improvements in several cognitive functions, especially among patients with greater pre-treatment impairments. ${ }^{32}$ Currently, there are a number of Phase 3 studies assessing the role of neuroprotectants within the context of PCI (NCT01553916, NCT00006349).

\section{$\mathrm{PCl}$ in the elderly}

Elderly patients are a rather interesting subgroup of SCLC patients. The proportion of elderly among all cases of SCLC has increased over the past 40 years. ${ }^{33}$ According to the Surveillance, Epidemiology, and End Results (SEER) database, elderly patients (>70 years) among all cases of SCLC increased from $23 \%$ in 1975 to $44 \%$ in 2010 . In general, elderly could be characterized by a lower performance status, higher comorbidity index, reduced organ function and less bone marrow reserve. Earlier studies have shown that elderly have significantly worse outcome compared to younger SCLC patients. ${ }^{34,35}$ Also, a large cohort study regarding therapeutic whole-brain irradiation reported significant toxicity such as neurocognitive dysfunction with memory loss in patients older than 70 years. ${ }^{36}$ In this context, elderly patients are the focus of research to reduce treatment-related toxicity of cranial irradiation and improve prognosis.

Historical studies reported that older patients ( $>60$ years) experience a greater risk of acute and chronic neurotoxicity after PCI, as well as patients treated with higher total dose (>30 Gy) or concurrent chemotherapy. ${ }^{37-39}$ Previous data from randomized trials and a meta-analysis ${ }^{4,6,11,18}$ included a varying number of older patients, which should be taken into consideration. 
The landmark study by Aupérin et al evaluated 987 patients from 7 studies focusing on oncologic outcome of PCI. ${ }^{4}$ Over $25 \%$ of all analyzed patients could be defined as elderly patients ( $\geq 65$ years). In the study by Le Péchoux et al, age ( $\leq 60 \mathrm{vs.}>60$ years) was a stratification factor for randomization. ${ }^{11}$ Unfortunately, the percentage of older patients in the analysis was not reported. Slotman et al included patients older than 75 years in their study, which represented over $7.5 \%$ of all participants. ${ }^{17}$

The SEER database analysis (1926 patients) published by Eaton et al focusing on elderly SCLC patients aged $\geq 75$ years confirmed application of PCI as an independent predictor of overall survival. ${ }^{40}$ However, the study did not report toxicity data for this patient subgroup.

A retrospective analysis of 658 LS SCLC patients from MD Anderson Cancer Center revealed that PCI conferred no survival benefit for patients aged $\geq 70$ years and explained it with an increased risk of death from other comorbidities and extracranial disease progression. ${ }^{36}$

In Takahashi et al's study, about $47 \%$ of patients in the PCI group and $46 \%$ in the observation group were aged 70 years or older. ${ }^{18}$ Takahashi et al found no overall survival benefit when administering PCI versus observation. Their suggestion is that PCI in patients with extensive-disease SCLC and confirmed absence of BM should be judged carefully because of the risk of declining cognitive function. Especially older patients appear to be at a higher risk of neurocognitive decline.

In summary, there is inconsistent data for PCI in elderly. The usage of PCI in this subgroup should be critically evaluated according to the stage of disease, treatment response to initial therapy, performance status, and existing general and neurological comorbidities. Data on the survival benefit of PCI in elderly are rather limited due to underrepresentation of this subgroup in prospective clinical trials. ${ }^{41}$

\section{$\mathrm{PCl}$ in resected early-stage SCLC}

Another relevant issue worth addressing is the issue of PCI in resected p-stage I SCLC.

The NCCN and European Society for Medical Oncology guidelines recommend adjuvant chemotherapy and PCI for early-stage SCLC, irrespective of primary treatment approach. We previously addressed this issue in two previous communications. ${ }^{42,43}$

Stahl et al demonstrated in an analysis of the National Cancer Data Base (NCDB) a surge in the utilization of surgery in 2004 vs. 2013 , up from $14.9 \%$ to $28.5 \%{ }^{44}$ The paucity of evidence suggests that surgery is feasible in clinical stage
I disease. However, careful selection of candidates including rigorous preoperative staging should be performed (positron emission tomography with 2-deoxy-2-[fluorine-18]fluoro-Dglucose integrated with computed tomography, endobronchial ultrasound-guided transbronchial needle aspiration and/or mediastinoscopy, comprehensive brain imaging using contrast-enhanced MRI) in order to ascertain stage I disease.

Current evidence supports adjuvant chemotherapy following surgery and is endorsed by another NCDB analysis by Yang et al in patients with pT1-2N0M0 disease, which demonstrated that these patients had inferior outcomes than those who underwent resection with adjuvant treatment. ${ }^{45}$

Regarding PCI in these patients, there is some evidence albeit retrospective data suggesting omission of PCI exclusively in the group of patients with p-stage I disease as various studies have shown a relatively low cumulative incidence of $\mathrm{BM}$, in the order of $10 \%{ }^{42}$ However, due to the paucity of data and expected discrepancies in the prevalence of BM, this recommendation does not extend to patients with clinical stage I disease.

\section{$\mathrm{PCl}$ in ES-SCLC}

The most intense debate recently has centered on universal delivery of PCI particularly in ES-SCLC. In an era in which there was a paucity of data regarding PCI delivery in these patients, the EORTC study by Slotman et al in 2007 demonstrated a reduction in risk of BM in the PCI group, cumulative risk of BM within 1 year of $14.6 \%$ and $40.4 \%$ in the PCI vs. control group, 1-year survival rate of $27.1 \%$ vs. $13.3 \%$ in the PCI vs. control group, association of PCI with an increase in median disease-free survival from 12.0 weeks to 14.7 weeks and an increase in median OS from 5.4 months to 6.7 months from randomization. ${ }^{6}$

However, the recently published Japanese study by Takahashi et al has sparked renewed debate. The study randomized 224 patients between 2009 and 2013 to PCI vs. observation. In the planned interim analysis on June 18, 2013, of the first 163 enrolled patients, Bayesian predictive probability of PCI, being superior to observation, was $0.011 \%$, resulting in early termination of the study due to futility. In the final analysis, median OS from randomization was 11.6 months in the PCI group vs. 13.7 months in the observation group. The authors concluded that PCI could be omitted in therapy responders under the premise that these patients be followed up comprehensively by serial brain imaging and radiotherapy be deferred till onset of BM. ${ }^{18}$

Both studies have been studied methodically and defining shortcomings have been leveled against both studies, 
which have been discussed extensively in various previous publications, ${ }^{18,46-49}$ particularly in the original publication by the Japanese group ${ }^{18}$ as well as a recently published review article by the same group. ${ }^{48}$

Most noteworthy was the mandated comprehensive brain imaging scheduling in the Japanese study, whereas in the EORTC study, CT/MRI of the brain was only performed in symptomatic patients. As such, only $29 \%$ of randomized patients in the EORTC study received brain imaging at diagnosis, and the number of patients who received repeat brain imaging prior to $\mathrm{PCI}$ is unclear.

Critics of the EORTC study partly attribute the survival benefit to the possible presence of subclinical BM before randomization, ascribing improved median OS to cranial irradiation. In addition, it is unclear what proportion of patients in the EORTC trial received platinum-based chemotherapy as mandated in the Japanese trial.

Moreover, various fractionation schedules at the discretion of the participating centers were adopted in the EORTC study with the predominant schedule being 20 Gy delivered in five fractions in 88/143 patients. By contrast, 25 Gy was universally delivered in 10 daily fractions in the Japanese trial.

However, the publication by Takahashi et al is not without its flaws. It is inevitable to question whether careful patient selection might have played a role as previously described in a previous communication by Slotman. ${ }^{46}$ On an average, participating centers enrolled only one patient per year and the high proportion of patients who received second-, thirdand fourth-line chemotherapy is incongruent with previously published data $^{49}$ and might reflect this assumption. In addition, in stark contrast to the EORTC trial, a higher proportion of patients received radiotherapy in the observation arm of the Japanese trial (25\% vs. 58\%). Le Péchoux et al described extensively similar 1-year survival rates in the subgroup analysis of ES-SCLC patients of the landmark PCI meta-analysis published in 1999. ${ }^{4,49}$ Differences in survival outcome between both studies were also attributed to several factors including eligibility criteria, patient selection, treatment regimens (chemotherapy and PCI fractionation), demographics as well as possibly related to host-related genetic differences in SCLC between Asian and non-Asian populations. $^{49}$

However, a retrospective study emanating from Germany demonstrated a stark improved median overall survival after PCI in ES-SCLC patients in comparison to the EORTC trial (12 vs. 6.7 months) and similar to the results of the Japanese trial. ${ }^{50}$ However, as the authors duly noted, according to institutional policy, the study population comprised a select group of patients with relatively good performance status (median ECOG 1) with exclusion of older patients and patients at high risk for cognitive decline.

\section{$\mathrm{PCl}$ vs. active $\mathrm{MRI}$ surveillance with access to stereotactic radiotherapy (SRT)}

Based on the conflicting results of the EORTC and Japanese trials, the NCCN softened its latest recommendation for PCI in ES-SCLC to "consider". However, in patients not receiving PCI, a comprehensive MRI surveillance program in accordance with the above-mentioned study is paramount.

Furthermore, there is uncertainty regarding use of SRT for brain relapse (single or multiple) in SCLC. There are some retrospective data supporting its use.

A secondary analysis of the Japanese trial noted that 25/54 (46\%) patients in the PCI arm received repeat radiotherapy with stereotactic radiosurgery (SRS) the preferred modality as is popularly adopted in the authors' country of origin. ${ }^{51}$

In a recent NCDB analysis of 5952 SCLC patients by Robin et al, upfront SRS was associated with improved median OS vs. upfront WBRT +/- SRS (10.8 vs. 7.1 months, HR $0.65,95 \%$ CI $0.55-0.75, p<0.001$ ), which persisted on multivariate analysis controlling for comorbidities, extracranial metastases, age, race/ethnicity and sex (HR 0.70, 95\% CI $0.60-0.81, p<0.001)$. However, the results must be interpreted with caution as the SRS arm was underpowered with 200 vs. 5752 patients in the WBRT arm. ${ }^{52}$

Also in patients with single brain metastasis, Bernhardt et al recently described prognostic factors and demonstrated in a retrospective analysis of 52 patients that, prior surgery, synchronous as opposed to metachronous metastasis and response to primary chemotherapy was associated with improved OS. ${ }^{53}$

Currently, a Phase 2 study in Germany comparing WBRT alone to SRS for SCLC patients is recruiting to this effect (NCT03297788) and might provide a basis for further investigation of this strategy within the ramifications of a Phase 3 study.

\section{Conclusion}

It is imminently paramount that the TNM classification according to the UICC 8th edition is universally adopted for SCLC.${ }^{54}$ Subsequently, survival benefit conferred by PCI in LS-SCLC could be further elucidated.

Since its initial proposal for SCLC in 1973 and recommendation in 1999, PCI has been the topic of recurring interest and debate. It is inevitable that we are slowly arriving at a defining crossroad as new data emanating from studies 
in the current MRI era and ubiquity of comprehensive brain imaging suggest that the modest survival benefit alluded to PCI may be less than previously anticipated, especially in ES-SCLC. Thus, further studies are urgently required to dispel these uncertainties.

However, the best strategy moving forward is probably integrating hippocampal avoidance and drug neuroprotection to minimize neuropsychological sequelae. Additionally, regarding the question of PCI in the elderly, there are some question marks that remain but, in our opinion, shared decision making is the pinnacle of patient-centered care. Subsequently, the next step, strictly hypothesis-generating would be evaluating possible dose de-escalation in elderly patients.

\section{Disclosure}

The authors report no conflicts of interest in this work.

\section{References}

1. Pietanza MC, Byers LA, Minna JD, Rudin CM. Small cell lung cancer: will recent progress lead to improved outcomes? Clin Cancer Res. 2015;21(10):2244-2255.

2. Siegel RL, Miller KD, Jemal A. Cancer statistics, 2015. CA Cancer J Clin. 2015;65(1):5-29.

3. Früh M, De Ruysscher D, Popat S, Crinò L, Peters S, Felip E; ESMO Guidelines Working Group. Small-cell lung cancer (SCLC): ESMO Clinical Practice Guidelines for diagnosis, treatment and follow-up. Ann Oncol. 2013;24(Suppl_6):vi99-vi105.

4. Aupérin A, Arriagada R, Pignon JP, et al. Prophylactic cranial irradiation for patients with small-cell lung cancer in complete remission. $N$ Engl J Med. 1999;341(7):476-484.

5. Kalemkerian GP, Akerley W, Bogner P, et al. Small cell lung cancer. J Natl Compr Cancer Netw. 2013;11(1):78-98.

6. Slotman B, Faivre-Finn C, Kramer G, et al; EORTC Radiation Oncology Group and Lung Cancer Group. Prophylactic cranial irradiation in extensive small-cell lung cancer. $N$ Engl J Med. 2007;357(7):664-672.

7. Slotman BJ, Mauer ME, Bottomley A, et al. Prophylactic cranial irradiation in extensive disease small-cell lung cancer: short-term health-related quality of life and patient reported symptoms - results of an International Phase III Randomized Controlled Trial by the EORTC radiation oncology and lung cancer groups. J Clin Oncol. 2009;27(1):78-84.

8. Käsmann L, Bolm L, Schild SE, Janssen S, Rades D. Neutrophil-tolymphocyte ratio predicts outcome in limited disease small-cell lung cancer. Lung. 2017;195(2):217-224.

9. Manapov F, Klautke G, Fietkau R. Prevalence of brain metastases immediately before prophylactic cranial irradiation in limited disease small cell lung cancer patients with complete remission to chemoradiotherapy: a single institution experience. J Thorac Oncol. 2008;3(6): 652-655.

10. Le Péchoux C, Dunant A, Senan S, et al; Prophylactic Cranial Irradiation (PCI) Collaborative Group. Standard-dose versus higher-dose prophylactic cranial irradiation (PCI) in patients with limited-stage small-cell lung cancer in complete remission after chemotherapy and thoracic radiotherapy (PCI 99-01, EORTC 22003-08004, RTOG 0212, and IFCT 99-01): a randomised clinical trial. Lancet Oncol. 2009;10(5): 467-474.

11. Schild SE, Foster NR, Meyers JP, et al; North Central Cancer Treatment Group. Prophylactic cranial irradiation in small-cell lung cancer: findings from a North Central Cancer Treatment Group Pooled Analysis. Ann Oncol. 2012;23(11):2919-2924.
12. Manapov F, Niyazi M, Gerum S, et al. Evaluation of the role of remission status in a heterogeneous limited disease small-cell lung cancer patient cohort treated with definitive chemoradiotherapy. BMC Cancer. 2016;16(1):216.

13. Rades D, Kaesmann L, Janssen S, Schild SE. A new score for estimating survival after definitive radiochemotherapy of limited disease small cell lung cancers. Lung. 2016;194(4):625-629.

14. Käsmann L, Janssen S, Rades D. Prognostic factors including the expression of Thyroid Transcription Factor 1 (TTF1) in patients irradiated for limited-disease small cell lung cancer. Anticancer Res. 2016;36(7):3499-3503.

15. Roengvoraphoj O, Eze C, Niyazi M, et al. Prognostic role of patient gender in limited-disease small-cell lung cancer treated with chemoradiotherapy. Strahlenther Onkol. 2017;193(2):150-155.

16. Eze C, Roengvoraphoj O, Niyazi M, et al. Treatment response and prophylactic cranial irradiation are prognostic factors in a real-life limited-disease small-cell lung cancer patient cohort comprehensively staged with cranial magnetic resonance imaging. Clin Lung Cancer. 2017;18(4):e243-e249.

17. Slotman BJ, van Tinteren H, Praag JO, et al. Use of thoracic radiotherapy for extensive stage small-cell lung cancer: a phase 3 randomised controlled trial. Lancet. 2015;385(9962):36-42.

18. Takahashi T, Yamanaka T, Seto T, et al. Prophylactic cranial irradiation versus observation in patients with extensive-disease small-cell lung cancer: a multicentre, randomised, open-label, phase 3 trial. Lancet Oncol. 2017;18(5):663-671.

19. Lee JS, Umsawasdi T, Lee YY, et al. Neurotoxicity in long-term survivors of small cell lung cancer. Int J Radiat Oncol Biol Phys. 1986;12(3):313-321.

20. Redmond KJ, Hales RK, Anderson-Keightly H, et al. Prospective study of hippocampal-sparing prophylactic cranial irradiation in limited-stage small cell lung cancer. Int J Radiat Oncol Biol Phys. 2017;98(3):603-611.

21. Gondi V, Tome WA, Marsh J, et al. Estimated risk of perihippocampal disease progression after hippocampal avoidance during wholebrain radiotherapy: safety profile for RTOG 0933. Radiother Oncol. 2010;95(3):327-331.

22. Gondi V, Pugh SL, Tome WA, et al. Preservation of memory with conformal avoidance of the hippocampal neural stem-cell compartment during whole-brain radiotherapy for brain metastases (RTOG 0933): a phase II multi-institutional trial. J Clin Oncol. 2014;32(34):3810-3816.

23. Lok BH, Ma J, Foster A, et al. Factors influencing the utilization of prophylactic cranial irradiation in patients with limited-stage small cell lung cancer. Adv Radiat Oncol. 2017;2(4):548-554.

24. Bennett BM, Wells JR, Panter C, Yuan Y, Penrod JR. The humanistic burden of small cell lung cancer (SCLC): a systematic review of healthrelated quality of life (HRQoL) literature. Front Pharmacol. 2017;8:339.

25. Wolfson AH, Bae K, Komaki R, et al. Primary analysis of a Phase II randomized trial radiation therapy oncology group (RTOG) 0212 : impact of different total doses and schedules of prophylactic cranial irradiation on chronic neurotoxicity and quality of life for patients with limited-disease small-cell lung cancer. Int J Radiat Oncol Biol Phys. 2011;81(1):77-84.

26. Sun A, Bae K, Gore EM, et al. Phase III trial of prophylactic cranial irradiation compared with observation in patients with locally advanced non-small-cell lung cancer: neurocognitive and quality-of-life analysis. J Clin Oncol. 2011;29(3):279-286.

27. Simó M, Vaquero L, Ripollés P, et al. Longitudinal brain changes associated with prophylactic cranial irradiation in lung cancer. JThorac Oncol. 2016;11(4):475-486.

28. Kalm M, Abel E, Wasling P, et al. Neurochemical evidence of potential neurotoxicity after prophylactic cranial irradiation. Int J Radiat Oncol Biol Phys. 2014;89(3):607-614.

29. Korkmaz Kirakli E, Oztekin O. Is hippocampal avoidance during whole-brain radiotherapy risky for patients with small-cell lung cancer? hippocampal metastasis rate and associated risk factors. Technol Cancer Res Treat. 2017;16(6):1202-1208. 
30. Liu M. Professor David Ball: prophylactic cranial irradiation (PCI) benefits patients with SCLC. Transl Lung Cancer Res. 2018;7(Suppl 1):S81-S82.

31. Brown PD, Pugh S, Laack NN, et al; Radiation Therapy Oncology Group (RTOG). Memantine for the prevention of cognitive dysfunction in patients receiving whole-brain radiotherapy: a randomized, doubleblind, placebo-controlled trial. Neuro Oncol. 2013;15(10):1429-1437.

32. Rapp SR, Case LD, Peiffer A, et al. Donepezil for irradiated brain tumor survivors: a Phase III randomized placebo-controlled clinical trial. J Clin Oncol. 2015;33(15):1653-1659.

33. Abdel-Rahman O. Changing epidemiology of elderly small cell lung cancer patients over the last 40 years; a SEER database analysis. Clin Respir J. 2018;12(3):1093-1099.

34. Ludbrook JJ, Truong PT, MacNeil MV, et al. Do age and comorbidity impact treatment allocation and outcomes in limited stage small-cell lung cancer? A community-based population analysis. Int J Radiat Oncol Biol Phys. 2003;55(5):1321-1330.

35. Rossi A, Maione P, Colantuoni G, et al. Treatment of small cell lung cancer in the elderly. Oncologist. 2005;10(6):399-411.

36. Farooqi AS, Holliday EB, Allen PK, Wei X, Cox JD, Komaki R. Prophylactic cranial irradiation after definitive chemoradiotherapy for limited-stage small cell lung cancer: Do all patients benefit? Radiother Oncol. 2017;122(2):307-312.

37. Johnson BE, Patronas N, Hayes W, et al. Neurologic, computed cranial tomographic, and magnetic resonance imaging abnormalities in patients with small-cell lung cancer: further follow-up of 6- to 13-year survivors. J Clin Oncol. 1990;8(1):48-56.

38. Shaw E, Su J, Eagan R, Jett J, Maksymiuk A, Deigert F. Prophylactic cranial irradiation in complete responders with small-cell lung cancer: analysis of the Mayo Clinic and North Central Cancer Treatment Group data bases. J Clin Oncol. 1994;12(11):2327-2332.

39. Glantz MJ, Choy H, Yee L. Prophylactic cranial irradiation in small cell lung cancer: rationale, results, and recommendations. Semin Oncol. 1997;24(4):477-483.

40. Eaton BR, Kim S, Marcus DM, et al. Effect of prophylactic cranial irradiation on survival in elderly patients with limited-stage small cell lung cancer. Cancer. 2013;119(21):3753-3760.

41. Damhuis RA, Senan S, Belderbos JS. Usage of prophylactic cranial irradiation in elderly patients with small-cell lung cancer. Clin Lung Cancer. 2018;19(2):e263-e267.

42. Eze C, Roengvoraphoj O, Manapov F. Prophylactic cranial irradiation in resected early stage small cell lung cancer. Int J Radiat Oncol. 2017;98(3):612-614.
43. Eze C, Roengvoraphoj O, Manapov F. Prophylactic cranial irradiation in resected small cell lung cancer: comprehensive staging, adjuvant chemotherapy, and strict stratification of pathological stage play a role. J Thorac Oncol. 2017;12(9):e137-e138.

44. Stahl JM, Corso CD, Verma V, et al. Trends in stereotactic body radiation therapy for stage I small cell lung cancer. Lung Cancer. 2017;103: 11-16.

45. Yang CF, Chan DY, Speicher PJ, et al. Role of adjuvant therapy in a population-based cohort of patients with early-stage small-cell lung cancer. J Clin Oncol. 2016;34(10):1057-1064.

46. Slotman BJ. Time to reconsider prophylactic cranial irradiation in extensive-stage small-cell lung cancer? Lancet Oncol. 2017;18(5): 566-567.

47. Manapov F, Eze C. Prophylactic cranial irradiation in small-cell lung cancer. Lancet Oncol. 2017;18(7):e366.

48. Nosaki K, Seto T, Shimokawa M, Takahashi T, Yamamoto N. Is prophylactic cranial irradiation (PCI) needed in patients with extensivestage small cell lung cancer showing complete response to first-line chemotherapy? Radiother Oncol. 2018;127(3):344-348.

49. Le Pechoux C, Botticella A, Levy A, Auperin A. Prophylactic cranial irradiation or no prophylactic cranial irradiation in metastatic small cell lung cancer: is it a relevant question once again? J Thorac Dis. 2017;9(11):4157-4161.

50. Bernhardt D, Adeberg S, Bozorgmehr F, et al. Nine-year experience: prophylactic cranial irradiation in extensive disease small-cell lung cancer. Clin Lung Cancer. 2017;18(4):e267-e271.

51. Akamatsu H, Takahashi T, Yamamoto N. Prophylactic cranial irradiation in small-cell lung cancer - Authors' reply. Lancet Oncol. 2017;18(7):e369.

52. Robin TP, Jones BL, Amini A, et al. Radiosurgery alone is associated with favorable outcomes for brain metastases from small-cell lung cancer. Lung Cancer. 2018;120:88-90.

53. Bernhardt D, Adeberg S, Bozorgmehr F, et al. Outcome and prognostic factors in single brain metastases from small-cell lung cancer. Strahlenther Onkol. 2018;194(2):98-106.

54. Goldstraw P, Chansky K, Crowley J, et al; International Association for the Study of Lung Cancer Staging and Prognostic Factors Committee Advisory Boards, and Participating Institutions. The IASLC lung cancer staging project: proposals for revision of the TNM stage groupings in the forthcoming (eighth) edition of the TNM classification for lung cancer. J Thorac Oncol. 2016;11(1):39-51.
Lung Cancer: Targets and Therapy

\section{Publish your work in this journal}

Lung Cancer: Targets and Therapy is an international, peer-reviewed, open access journal focusing on lung cancer research, identification of therapeutic targets and the optimal use of preventative and integrated treatment interventions to achieve improved outcomes, enhanced survival and quality of life for the cancer patient. Specific topics covered in the journal include: Epidemiology, detection and screening Cellular research and biomarkers; Identification of biotargets and agents with novel

Submit your manuscript here: https://www.dovepress.com/lung-cancer-targets--therapy-journa mechanisms of action; Optimal clinical use of existing anticancer agents, including combination therapies; Radiation and surgery; Palliative care; Patient adherence, quality of life, satisfaction; Health economic evaluations. The manuscript management system is completely online and includes a very quick and fair peer-review system. Visit http://www.dovepress.com/testimonials.php to read real quotes from published authors. 